\title{
RECONSTRUCTION IN MALIGNANT TUMORS OF THE CALF AFTER TUMORAL RESECTION IN CHILDREN
}

doi: 10.2478/rojost-2018-0027

C. Vlad ${ }^{1}$, L. Dobre ${ }^{1}$, C. Vreme ${ }^{1}$, Ș.A. Hamei ${ }^{1}$, Ş. Gavriliu'1,2

${ }^{1}$ "M.S. Curie" Children Clinical Emergency Hospital, Bucharest, Romania

2"Carol Davila" University of Medicine and Pharmacy, Bucharest, Romania

Purpose. Evaluation of reconstructive procedures after oncologic resection of malignant bone tumors of the calf in children.

Materials and methods. The study contains 4 patients with ages between 5 and 18 years, 2 of the cases being diagnosed with osteosarcoma, respectively 2 with Ewing sarcoma of the tibia. In 3 of the cases, the surgical treatment consisted of en bloc tumoral resection and reconstruction with metal acrylic spacer and, in the $4^{\text {th }}$ case, the tumoral resection was followed by endoprosthetic replacement. In all cases, the surgical treatment was preceded by biopsy and chemotherapy.

Results. In 3 of the patients we used a gastrocnemius flap and one patient needed a negative pressure dressing for 11 days after surgery. The follow-up varied between 8 and 17 months after surgery. The patient who underwent endoprosthetic replacement was immobilized in a mobile orthosis and partial weight bearing at 2 weeks PO.

The patient who underwent endoprosthetic replacement needed a surgical revision at 10 months $\mathrm{PO}$ consisting in the reinsertion of a locking screw of the patellar tendon fixation device. 2 of the presented cases are waiting for replacement of the metal acrylic spacer with a definitive reconstruction procedure, 1 case is proposed for amputation.

Conclusions. Malignant bone tumors of the calf in children represent a pathology with high complexity giving the possible complications that can occur, a redoubtable risk being implant loss due to lack of coverage. The discussion remains open regarding the right time for definitive surgical procedure with modular endoprosthesis in children, and other reconstructive techniques available in these cases.

Keywords: osteosarcoma, Ewing sarcoma, biopsy, reconstruction 